\title{
PARTICLE PHYSICS-FUTURE DIRECTIONS
}

\author{
Chris Quigg*, Fermi National Accelerator Laboratory, Batavia, IL 60510, USA
}

\section{Abstract}

Wonderful opportunities await particle physics over the next decade, with the coming of the Large Hadron Collider at CERN to explore the $1-\mathrm{TeV}$ scale (extending efforts at LEP and the Tevatron to unravel the nature of electroweak symmetry breaking) and many initiatives to develop our understanding of the problem of identity: what makes a neutrino a neutrino and a top quark a top quark. Here I have in mind the work of the $B$ factories and the Tevatron collider on $C P$ violation and the weak interactions of the $b$ quark; the wonderfully sensitive experiments at Brookhaven, CERN, Fermilab, and Frascati on CP violation and rare decays of kaons; the prospect of definitive accelerator experiments on neutrino oscillations and the nature of the neutrinos; and a host of new experiments on the sensitivity frontier. We might even learn to read experiment for clues about the dimensionality of spacetime. If we are inventive enough, we may be able to follow this rich menu with the physics opportunities offered by a linear collider and a (muon storage ring) neutrino factory. I expect a remarkable flowering of experimental particle physics, and of theoretical physics that engages with experiment. I describe some of the great questions before us and the challenges of providing the instruments that will be needed to define them more fully and-eventually-to answer them.

\section{PARTICLE PHYSICS AT THE MILLENNIUM}

The physics curriculum in the 1898-99 University of Chicago catalogue begins with a very Victorian preface [1]:

\begin{abstract}
"While it is never safe to affirm that the future of the Physical Sciences has no marvels in store even more astonishing than those of the past, it seems probable that most of the grand underlying principles have been firmly established and that further advances are to be sought chiefly in the rigorous application of these principles to all the phenomena which come under our notice.... An eminent physicist has remarked that the future truths of Physical Science are to be looked for in the sixth place of decimals."
\end{abstract}

As the ink was drying on these earnest words, Röntgen discovered $x$ rays and published the epoch-making radiograph of his wife's hand, Becquerel and the Curies explored radioactivity, Thomson discovered the electron and showed that the "uncuttable" atom had parts, and Planck noted

\footnotetext{
*quigg@fnal.gov
}

that anomalies in the first place of the decimals required a wholesale revision of the laws of Nature.

We have the benefit of a century of additional experience and insight, but we are not nearly so confident that we have uncovered "most of the grand underlying principles." Indeed, while we celebrate the insights codified in the standard model of particle physics and look forward to resolving its puzzles, we are increasingly conscious of how little of the physical universe we have experienced. $\mathrm{Fu}$ ture truths are still to be found in precision measurements, but the century we are leaving has repeatedly shown that Nature's marvels are not limited by our imagination. Exploration can yield surprises that completely change what we think about-and how we think.

We base our understanding of physical phenomena on the identification of a few constituents that seem elementary at the current limits of resolution of about $10^{-18} \mathrm{~m}$, and a few fundamental forces. The constituents are the pointlike quarks $\left\{(u, d)_{L},(c, s)_{L},(t, b)_{L}\right\}$ and leptons $\left\{\left(\nu_{e}, e\right)_{L},\left(\nu_{\mu}, \mu\right)_{L},\left(\nu_{\tau}, \tau\right)_{L}\right\}$, with strong, weak, and electromagnetic interactions specified by $S U(3)_{c} \otimes S U(2)_{L} \otimes$ $U(1)_{Y}$ gauge symmetries.

The electroweak theory is founded on the weak-isospin symmetry embodied in the quark and lepton doublets and weak-hypercharge phase symmetry, plus the idealization that neutrinos are massless. ${ }^{1}$ In its simplest form, with the electroweak gauge symmetry broken by the Higgs mechanism, the $S U(2)_{L} \otimes U(1)_{Y}$ theory has scored many qualitative successes: the prediction of neutral-current interactions, the necessity of charm, the prediction of the existence and properties of the weak bosons $W^{ \pm}$and $Z^{0}$. Over the past ten years, in great measure due to the beautiful experiments carried out at the $Z$ factories at CERN and SLAC, precision measurements have tested the electroweak theory as a quantum field theory, at the one-permille level $[3,4,5]$. Last year, our colleagues working at LEP made a heroic push to discover the Higgs boson 2000 [6]. The search will intensify again in a few years at the Tevatron and the Large Hadron Collider.

The quark model of hadron structure and the parton model of hard-scattering processes have such pervasive influence on the way we conceptualize particle physics that quantum chromodynamics, the theory of strong interactions that underlies both, sometimes is taken for granted. QCD is a remarkably simple, successful [7], and rich theory of the strong interactions [8]. The perturbative regime of QCD exists, thanks to the crucial property of asymptotic freedom, and describes many phenomena in quantitative detail. The strong-coupling regime controls hadron struc-

${ }^{1}$ For surveys of the electroweak theory, with references, see Ref. [2]. 
ture and gives us our best information about quark masses. Unfamiliar régimes of high density and high temperature contain riches we have only begun to explore.

\section{WHAT WE NEED TO KNOW}

This concise statement of the standard model invites us to consider the agenda of particle physics today under a few broad rubrics.

Elementarity. Are the quarks and leptons structureless, or will we find that they are composite particles with internal structures that help us understand the properties of the individual quarks and leptons?

Symmetry. One of the most powerful lessons of the modern synthesis of particle physics is that (local) symmetries prescribe interactions. Our investigation of symmetry must address the question of which gauge symmetries exist (and, eventually, why). We have learned to seek symmetry in the laws of Nature, not (necessarily) in the consequences of those laws. Accordingly, we must understand how the symmetries are hidden from us in the world we inhabit. For the moment, the most urgent problem in particle physics is to complete our understanding of electroweak symmetry breaking by exploring the $1-\mathrm{TeV}$ scale. This is the business of the experiments at LEP2, the Tevatron Collider, and the Large Hadron Collider.

Unity. In the sense of developing explanations that apply not to one individual phenomenon in isolation, but to many phenomena in common, unity is central to all of physics, and indeed to all of science. At this moment in particle physics, our quest for unity takes several forms.

First, we have the fascinating possibility of gauge coupling unification, the idea that all the interactions we encounter have a common origin and thus a common strength at suitably high energy.

Second, there is the imperative of anomaly freedom in the electroweak theory, which urges us to treat quarks and leptons together, not as completely independent species. Both of these ideas are embodied, of course, in unified theories of the strong, weak, and electromagnetic interactions, which imply the existence of still other forces- to complete the grander gauge group of the unified theoryincluding interactions that change quarks into leptons.

The third aspect of unity is the idea that the traditional distinction between force particles and constituents might give way to a unified understanding of all the particles. The gluons of QCD carry color charge, so we can imagine quarkless hadronic matter in the form of glueballs. Beyond that breaking down of the wall between messengers and constituents, supersymmetry relates fermions and bosons.

Finally, we desire a reconciliation between the pervasive outsider, gravity, and the forces that prevail in the quantum world of our everyday laboratory experience.

Identity. We do not understand the physics that sets quark masses and mixings. Although we are testing the idea that the phase in the quark-mixing matrix lies behind the ob- served $\mathrm{CP}$ violation, we do not know what determines that phase. The accumulating evidence for neutrino oscillations presents us with a new embodiment of these puzzles in the lepton sector. At bottom, the question of identity is very simple to state: What makes an electron and electron, and a top quark a top quark?

Topography. "What is the dimensionality of spacetime?" tests our preconceptions and unspoken assumptions. It is given immediacy by recent theoretical work. For its internal consistency, string theory requires an additional six or seven space dimensions, beyond the $3+1$ dimensions of everyday experience. Until recently it has been presumed that the extra dimensions must be compactified on the Planck scale, with a stupendously small compactification radius $R \simeq M_{\text {Planck }}^{-1}=1.6 \times 10^{-35} \mathrm{~m}$. Part of the vision of string theory is that what goes on in even such tiny curled-up dimensions does affect the everyday world: excitations of the Calabi-Yau manifolds determine the fermion spectrum. ${ }^{2}$

We have recognized recently that Planck-scale compactification is not-according to what we can establishobligatory, and that current experiment and observation admit the possibility of dimensions not navigated by the strong, weak, and electromagnetic interactions that are almost palpably large. A whole range of new experiments will help us explore the fabric of space and time, in ways we didn't expect just a few years ago [10].

\section{A DECADE OF DISCOVERY AHEAD}

Over the next decade, we may look forward to an avalanche of experimental results that have the potential to change our view of the fundamental particles and their interactions in very dramatic ways. A special preoccupation for me is the search and study of the Higgs boson; this is really shorthand for a thorough exploration of the $1-\mathrm{TeV}$ scale, which will elucidate the mechanism of electroweak symmetry breaking. We can also expect wonderful progress in flavor physics: the detailed study of CP violation in the $B$ system, dramatically increased sensitivity in the exploration of rare decays of $K$ and $D$ mesons, and pinning down the nature of neutrino oscillations. And maybe we will at last see a CP-violating permanent electric dipole moment of the neutron. Run II of the Tevatron will give us our first opportunity to use the top quark as a tool, and not only as an object of desire. Although the interpretation of heavy-ion collisions at RHIC and the LHC promises to be challenging, the heavy-ion colliders offer a real chance to discover new phases of matter and enrich our understanding of QCD. On many fronts, we are taking dramatic steps in energy and sensitivity that will help us explore: extra dimensions, new dynamics, supersymmetry, and new kinds of forces and constituents might show themselves. (I'm conflicted about whether I'd like to see them all at once, or in easy-to-understand installments!)

Experiments that use natural sources also hold great

\footnotetext{
${ }^{2}$ For a gentle introduction to the goals of string theory, see Ref. [9].
} 
promise for the decade ahead. We suspect that the detection of proton decay is only a few orders of magnitude away in sensitivity. Astronomical observations should tell us what kinds of matter and energy make up the universe. Indeed, the whole complex of experiments and observations we call astro/cosmo/particle physics should enjoy a golden age.

Now, the decade of discovery won't happen automatically. Many of our goals are difficult, and timely success is in doubt for many experiments. We must push hard to prepare the instruments, and get to the answers.

The glorious future of new machines and new experiments that lies beyond the established program also won't happen by itself. We have, I think, come to the collective realization that we must do more to prepare alternative futures by creating a rich and organic program of accelerator research. We're also challenged by our success: the scope of our science has grown, but funding has not. Within our own extended family and beyond, we must do more to convey the urgency and importance of the new scientific opportunities, and fashion a program that we can carry out that includes the right measure of scale diversity to ensure a healthy intellectual ecosystem.

\section{THE ORIGINS OF MASS}

A key aspect of the problem of identity is the origin of mass. In fact, we know the challenge of explaining many different kinds of mass. The masses of the hadrons are (in principle, and with increasing precision in practice) understood from QCD in terms of the energy stored to confine a color-singlet configuration of quarks in a small volume $[11,12]$. This is a remarkable achievement. We also have an excellent understanding of the masses of the electroweak gauge bosons $W^{ \pm}$and $Z^{0}$ as consequences of electroweak symmetry breaking, in terms of a single weak mixing parameter, $\sin ^{2} \theta_{W} \cdot{ }^{3}$ At tree level in the electroweak theory, we have

$$
\begin{aligned}
M_{W}^{2} & =g^{2} v^{2} / 2=\pi \alpha / G_{F} \sqrt{2} \sin ^{2} \theta_{W}, \\
M_{Z}^{2} & =M_{W}^{2} / \cos ^{2} \theta_{W},
\end{aligned}
$$

with the electroweak scale $v=\left(G_{F} \sqrt{2}\right)^{-\frac{1}{2}} \approx 246 \mathrm{GeV}$.

When we get to the question of quark and (charged) lepton masses, however, our understanding is considerably more primitive. For each of these, we require not just the scale of electroweak symmetry breaking, but a distinct and apparently arbitrary Higgs-fermion-antifermion Yukawa coupling to reproduce the fermion mass. In the electroweak theory, the value of each quark or chargedlepton mass is set by a new, unknown, Yukawa coupling. Taking the electron as a prototype, we define the lefthanded doublet and right-handed singlet

$$
\mathrm{L}=\left(\begin{array}{c}
\nu_{e} \\
e
\end{array}\right)_{L}, \quad \mathrm{R} \equiv e_{R}
$$

\footnotetext{
${ }^{3}$ Although for the moment we take the weak mixing parameter from experiment, we understand how it arises in a unified theory. Indeed, in a unified theory we can hope to understand the parameter $\Lambda_{\mathrm{QCD}}$ that sets the scale of the hadron masses.
}

Then the Yukawa term in the electroweak Lagrangian is

$$
\mathcal{L}_{\text {Yukawa }}^{(e)}=-\zeta_{e}\left[\overline{\mathrm{R}}\left(\varphi^{\dagger} \mathrm{L}\right)+(\overline{\mathrm{L}} \varphi) \mathrm{R}\right],
$$

where $\varphi$ is the Higgs field, so that the electron mass is $m_{e}=\zeta_{e} v / \sqrt{2}$. For neutrinos, which may be their own antiparticles, there are still more possibilities for new physics to enter. Inasmuch as we do not know how to calculate the fermion Yukawa couplings $\zeta_{f}$, I believe that we should consider the sources of all fermion masses as physics beyond the standard model.

The values of the Yukawa couplings are vastly different for different fermions: for the top quark, $\zeta_{t} \approx 1$, for the electron $\zeta_{e} \approx 3 \times 10^{-6}$, and if the neutrinos have Dirac masses, presumably $\zeta_{\nu} \approx 10^{-10} .^{4}$ What accounts for the range and values of the Yukawa couplings? Our best hope until now has been the suggestion from unified theories that the pattern of fermion masses simplifies on high scales. The classic intriguing prediction of the $S U(5)$ unified theory involves the masses of the $b$ quark and the $\tau$ lepton, which are degenerate at the unification point for a simple pattern of spontaneous symmetry breaking. The different running of the quark and lepton masses to low scales then leads to the prediction $m_{b} \approx 3 m_{\tau}$, in suggestive agreement with what we know from experiment [13].

"Large" extra dimensions present us with new ways to think about the exponential range of Yukawa couplings. If the standard-model brane has a small thickness, the wave packets representing different fermion species might have different locations within the extra dimension $[14,15]$. On this picture, the Yukawa couplings measure the overlap in the extra dimensions of the left-handed and right-handed fermion wave packets and the Higgs field, presumed pervasive. Exponentially large differences might then arise from small offsets in the new coordinate(s). True or not, it is a mind-expanding way to look at an important problem.

\section{GRAVITY AND EXTRA DIMENSIONS}

It is entirely natural to neglect gravity in most particlephysics applications, because the coupling of a graviton $\mathcal{G}$ to a particle is tiny, generically of order $\left(E / M_{\text {Planck }}\right)$ where $E$ is a typical energy scale of the problem. Thus, for example, we expect the branching fraction $B(K \rightarrow \pi \mathcal{G}) \sim$ $\left(M_{K} / M_{\text {Planck }}\right)^{2} \sim 10^{-38}$. And yet we cannot put gravity entirely out of our minds, even if we restrict our attention to standard-model interactions at attainable energies.

The great gap between the electroweak scale of about $10^{3} \mathrm{GeV}$ and the Planck scale of about $10^{19} \mathrm{GeV}$ gives rise to the hierarchy problem of the electroweak theory [16]: how to protect the Higgs-boson mass from quantum corrections that explore energies up to $M_{\text {Planck }}$. The conventional approach to the hierarchy problem has been to ask why the electroweak scale (and the mass of the Higgs boson) is so much smaller than the Planck scale. Framing the issue this way leads us to change the electroweak

\footnotetext{
${ }^{4}$ I am quoting the values of the Yukawa couplings at a low scale typical of the masses themselves.
} 
theory to include supersymmetry, or technicolor, or some other extension. Over the past few years, we have begun instead to ask why gravity is so weak. This question motivates us to consider changing gravity to understand why the Planck scale is so large [17]. Now, elegant experiments that study details of Casimir and van der Waals forces tell us that gravitation closely follows the Newtonian force law down to distances on the order of $0.3 \mathrm{~mm}$ [18], which corresponds to an energy scale of only about $10^{-12} \mathrm{GeV}$ ! At shorter distances (higher energies), the constraints on deviations from Newton's inverse-square force law deteriorate rapidly, so nothing prevents us from considering changes to gravity even on a small but macroscopic scale.

One way to change the force law is to imagine that gravity can propagate into extra dimensions. To respect the stronger constraints on the behavior of the standard-model interactions, we suppose that the $S U(3)_{c} \otimes S U(2)_{L} \otimes$ $U(1)_{Y}$ gauge fields, plus needed extensions, reside on $3+1$-dimensional branes, not in the extra dimensions.

How does this hypothesis change the picture? The dimensional analysis (Gauss's law, if you like) that relates Newton's constant to the Planck scale changes. If gravity propagates in $n$ extra dimensions with radius $R$, then

$$
G_{\text {Newton }} \sim M_{\text {Planck }}^{-2} \sim M^{\star-n-2} R^{-n},
$$

where $M^{\star}$ is gravity's true scale. Notice that if we boldly take $M^{\star}$ to be as small as $1 \mathrm{TeV} / c^{2}$, then the radius of the extra dimensions is required to be smaller than about $1 \mathrm{~mm}$, for $n \geq 2$. If we use the four-dimensional force law to extrapolate the strength of gravity from low energies to high, we find that gravity becomes as strong as the other forces on the Planck scale. If the force law changes at an energy $1 / R$, as the large-extra-dimensions scenario suggests, then the forces are unified at lower energy $M^{\star}$. What we know as the Planck scale is then a mirage that results from a false extrapolation: treating gravity as four-dimensional down to arbitrarily small distances, when in fact-or at least in this particular fiction-gravity propagates in $3+n$ spatial dimensions. The Planck mass is an artifact, given by $M_{\text {Planck }}=M^{\star}\left(M^{\star} R\right)^{n / 2}$. If the true scale of gravity were close to $M_{H}$, the hierarchy problem would recede.

\section{$6 \nu$ OSCILLATION NEWS}

The science that grew into particle physics began with found beams - the emanations from naturally occurring radioactive substance and the cosmic rays - and found beams still provide us with important windows on the universe. One of the great scientific detective stories of the recent past is the developing case for neutrino oscillations: the evidence that neutrinos produced as one flavor $\left(\nu_{e}, \nu_{\mu}\right.$, or $\left.\nu_{\tau}\right)$ actually morph into other flavors. Long known as a theoretical possibility, neutrino oscillation is now all but established by the Super-Kamiokande experiment's observation of an up-down asymmetry in the flux of muon neutrinos produced by the interaction of cosmic rays in the atmosphere [19]. By far the most graceful interpretation is that $\nu_{\mu}$ produced on the other side of the Earth oscillate during flight in significant numbers into $\nu_{\tau}$.

Just as PAC2001 convened, the Sudbury Neutrino Observatory added an important new element to our understanding of the longstanding puzzle of the solar neutrino deficit [20]. SNO reports an impressively precise measurement of the solar neutrino charged-current cross section on the heavy-water target that serves as their water-Cherenkov detector. The measured rate implies a $\nu_{e}$ flux

$$
\phi_{\mathrm{SNO}}^{\mathrm{CC}}\left(\nu_{e}\right)=1.75 \pm 0.07_{-0.11}^{+0.12} \pm 0.05 \times 10^{6} \mathrm{~cm}^{-2} \mathrm{~s}^{-1}
$$

where the uncertainties are statistical, systematic, and theoretical. They have also measured the solar neutrino elastic $\left(\nu_{x} e\right)$ cross section with limited precision, and extracted from it the flux of solar neutrinos of all active flavors,

$$
\phi_{\mathrm{SNO}}^{\mathrm{ES}}\left(\nu_{x}\right)=2.39 \pm 0.34_{-0.14}^{+0.16} \times 10^{6} \mathrm{~cm}^{-2} \mathrm{~s}^{-1} .
$$

The SNO experimenters are in the right place at the right time, because the Super-K experiment has already given a very precise measurement of the solar neutrino flux from elastic $\left(\nu_{x} e\right)$ scattering [21],

$$
\phi_{\mathrm{SK}}^{\mathrm{ES}}\left(\nu_{x}\right)=2.32 \pm 0.03_{-0.07}^{+0.08} \times 10^{6} \mathrm{~cm}^{-2} \mathrm{~s}^{-1} .
$$

The difference between the flux of active neutrinos and the flux of electron neutrinos,

$$
\phi_{\mathrm{SK}}^{\mathrm{ES}}\left(\nu_{x}\right)-\phi_{\mathrm{SNO}}^{\mathrm{CC}}\left(\nu_{e}\right)=0.57 \pm 0.17 \times 10^{6} \mathrm{~cm}^{-2} \mathrm{~s}^{-1}
$$

demonstrates at $3.3 \sigma$ that active neutrinos other than $\nu_{e}$, namely $\nu_{\mu}$ and $\nu_{\tau}$, arrive at Earth. Since the nuclear processes that power the Sun yield only $\nu_{e}$, this new result rules in favor of neutrino oscillations as the explanation for the solar neutrino puzzle.

\section{THE QUEST FOR NEW TOOLS}

Although theoretical speculation and synthesis is valuable and necessary, we cannot advance without new observations. The experimental clues needed to answer today's central questions can come from experiments at highenergy accelerators, experiments at low-energy accelerators and nuclear reactors, experiments with found beams, and deductions from astrophysical measurements. Past experience, our intuition, and the current state of particle theory all point to an indispensable role for accelerator experiments.

The opportunities for accelerator science and technology are multifaceted and challenging, and offer rich rewards for particle physics. One line of attack consists in refining known technologies to accelerate and collide the traditional projectiles-electrons, protons, and their antiparticlespushing the frontiers of energy, sensitivity, and precise control. The new instruments might include brighter proton sources; very-high-luminosity $e^{+} e^{-}$"factories" for $B, \tau$ / charm, $\phi, \ldots$; cost-effective hadron colliders beyond the LHC at CERN; and $e^{+} e^{-}$linear colliders. 
A second approach entails the development of exotic acceleration technologies for standard particles: electrons, protons, and their antiparticles. We don't yet know what instruments might result from research into new acceleration methods, but it is easy to imagine dramatic new possibilities for particle physics, condensed matter physics, applied science, medical diagnostics and therapies, and manufacturing, as well as a multitude of security applications.

A third path involves the exploration of exotic particles for accelerators and colliders to expand the experimenter's armamentarium. Muon storage rings for neutrino factories, $\mu^{+} \mu^{-}$colliders and $\gamma \gamma$ colliders are all under active investigation, and each of these would bring remarkable new possibilities for experiment.

Finally, let us note the continuing importance of enabling technologies: developing or domesticating new materials, new construction methods, new instrumentation, and new active controls.

To a very great extent, the progress of particle physics has been paced by progress in accelerator science and technology. A renewed commitment to accelerator research and development [22] will ensure a vigorous intellectual life for accelerator science and lead to important new tools for particle physics and beyond.

\section{CONCLUDING REMARKS}

In the midst of a revolution in our conception of Nature, we confront many fundamental questions about our world of diversity and change. Are the quarks and leptons elementary or composite? What are the symmetries of Nature, and how are they hidden from us? Will we find new forms of matter, like the superpartners suggested by supersymmetry? Will we find additional fundamental forces? What makes an electron an electron and a top quark a top quark? What is the dimensionality of spacetime, what is its shape?

These are themselves great questions and, in the usual way of science, answering them can lead us toward the answers to yet broader and more cosmic questions. As we contemplate the far-reaching understanding we can hope to create together, it is inspiring to remember the words Michael Faraday recorded in his Research Notes of 19th March 1849:

Nothing is too wonderful to be true, if it be consistent with the laws of nature ... Experiment is the best test ...

\section{ACKNOWLEDGMENT}

Fermilab is operated by Universities Research Association Inc. under Contract No. DE-AC02-76CH03000 with the United States Department of Energy.

\section{REFERENCES}

[1] Treiman, S., The Odd Quantum, (Princeton University Press, Princeton, 1999).
[2] Quigg, C., Acta Phys. Polon. B30, 2145 (1999);“"The Electroweak Theory," FERMILAB-CONF-01-001-T, in Flavor Physics for the Millennium (TASI 2000), Boulder, Colorado, 4-30 June 2000, edited by J. L. Rosner (World Scientific, Singapore, 2001), pp. 3-67.

[3] Sirlin, A., "Ten Years of Precision Electroweak Physics," in Proc. of the 19th Intl. Symp. on Photon and Lepton Interactions at High Energy LP99, ed. J. A. Jaros and M. E. Peskin, Int. J. Mod. Phys. A 15S1, 398 (2000) [eConf C990809, 398 (2000)], hep-ph/9912227.

[4] Swartz, M., "Precision Electroweak Physics at the $Z$," in Proc. of the 19th Intl. Symp. on Photon and Lepton Interactions at High Energy LP99, ed. J. A. Jaros and M. E. Peskin, Int. J. Mod. Phys. A 15S1, 307 (2000) [eConf C990809, 307 (2000)], hep-ex/9912026.

[5] Charlton, D., "Experimental tests of the standard model," to appear in the proceedings of International Europhysics Conference on High-Energy Physics (HEP 2001), Budapest, Hungary, 12-18 Jul 2001, hep-ex/0110086.

[6] LEP Working Group for Higgs boson searches, "Search for the standard model Higgs boson at LEP," hep-ex/0107029.

[7] Quigg, C., "The state of the standard model," hep$\mathrm{ph} / 0001145$, in Physics Potential and Development of Muon Colliders and Neutrino Factories, edited by David B. Cline, AIP Conference Proceedings 542 (American Institute of Physics, Melville, NY, 2000), pp. 3-28.

[8] Wilczek, F., Nucl. Phys. A663, 3 (2000).

[9] Greene, B., The Elegant Universe (Norton, NY, 1999).

[10] For a popular account see Abel, S. and MarchRussell, J., "The search for extra dimensions," Physics World 13, (11) 39 (November 2000), available at http://physicsweb.org/article/world/13/11/9.

[11] Wilczek, F., Phys. Today 52, (11) 11 (November 1999).

[12] Aoki, S., et al. (CP-PACS Collaboration), Phys. Rev. Lett. 84, 238 (2000).

[13] Buras, A. J., Ellis, J. R., Gaillard, M. K., and Nanopoulos, D. V., Nucl. Phys. B135, 66 (1978).

[14] Arkani-Hamed, N., and Schmaltz, M., Phys. Rev. D61, 033005 (2000).

[15] Mirabelli, E. A., and Schmaltz, M., Phys. Rev. D61, 113011 (2000).

[16] Gildener, E., Phys. Rev. D14, 1667 (1976); Weinberg, S., Phys. Lett. 82B, 387 (1979).

[17] Among the seminal papers, see Antoniadis, I., Phys. Lett. B246, 377 (1990); Lykken, J. D., Phys. Rev. D54, 3693 (1996); Arkani-Hamed, N., Dimopoulos, S., and Dvali, G., Phys. Lett. B429, 263 (1998).

[18] Hoyle, C. D., et al., Phys. Rev. Lett. 86, 1418 (2001).

[19] Toshito, T. [SuperKamiokande Collaboration], "SuperKamiokande atmospheric $\nu$ results," hep-ex/0105023.

[20] Ahmad, Q. R., et al. [SNO Collaboration], Phys. Rev. Lett. 87, 071301 (2001).

[21] Fukuda, S., et al. [SuperKamiokande Collaboration], Phys. Rev. Lett. 86, 5651 (2001).

[22] Chao, Alexander, et al., "2001 Snowmass Accelerator R\&D Report," prepared by the Division of Physics of Beams of the American Physical Society, available at http://www.hep.anl.gov/pvs/dpb/Snowmass.pdf. 